\section{Rede de frio para a conservação de vacinas em unidades públicas do município de São Paulo: conhecimento e prática}

\section{Vaccine storage cold chain in public health units of the City of São Paulo: knowledge and practice}

\section{Resumo}

Para assegurar a eficácia das vacinas utilizadas no Programa de Imunização é fundamental a manutenção da rede de frio. Este estudo tem o objetivo de avaliar o conhecimento e a prática adotados na conservação das vacinas nas unidades públicas do município de São Paulo. Foram enviados formulários a 390 salas com atividades de rotina e 80 foram selecionadas para uma entrevista com um profissional e observação do refrigerador. Um indicador foi construído para sintetizar os resultados, mediante a atribuição de pontos a itens comuns dos instrumentos utilizados, estabelecendo-se a classificação arbitrária de conhecimento/cumprimento SUFICIENTE, REGULAR e INSUFICIENTE. Sessenta por cento das unidades responderam o formulário, 80 salas foram visitadas e 90 refrigeradores foram observados. Resultados SUFICIENTES foram obtidos por $66 \%$ das observações, $55 \%$ das entrevistas e $53 \%$ dos formulários. O treinamento em sala de vacina influenciou positivamente a maior pontuação das observações. A concordância entre os instrumentos foi baixa, proporcionando a reflexão sobre a maneira como as informações da conservação de vacinas são transmitidas e a necessidade da supervisão sistemática para correção das falhas detectadas.

Palavras-chave: Vacinas. Refrigeração. Programas de Imunização. Serviços de saúde comunitária.

\section{Clelia Maria Sarmento de Souza Aranda ${ }^{1}$ José Cássio de Moraes ${ }^{2}$ \\ 'Divisão de Imunização, Centro de Vigilância Epidemiológica, Secretaria de Estado da Saúde de São Paulo. \\ ${ }^{2}$ Departamento de Medicina Social, Faculdade de Ciências Médicas da Santa Casa de São Paulo.}

Estudo apoiado pela Coordenadoria de Controle de Doenças/SES-SP.

Correspondência: Clelia Maria Sarmento. Divisão de Imunização/CVE-SES/SP. R. Dr. Enéas de Carvalho Aguiar, 188 - $6^{\circ}$ andar - sl 608 - CEP 05403-000 - São Paulo, SP.

E-mail: cmaranda@saude.sp.gov.br 


\section{Abstract}

The cold chain is essential to assure the efficacy of the vaccines used in immunization programs. This study aimed to assess the knowledge and the practice adopted in vaccine storage in public units of the city of São Paulo. Forms were sent to 390 health units with custom activities, and 80 were selected for a visit and an interview with a health worker and for observation of the refrigerator. An indicator was built to summarize results, and scores were given to common items of the tools utilized, establishing an arbitrary classification of SUFFICIENT, REGULAR and INSUFFICIENT knowledge/accomplishment. Sixty percent of the units answered the form, all the selected units were visited, and 90 refrigerators were observed. SUFFICIENT results were achieved by $66 \%$ observations, $55 \%$ interviews, and $53 \%$ formularies. Training in vaccine units influenced positively higher scores in observations. Agreement among tools was low, leading to considerations on the way in which vaccines storage guidelines are conveyed, along with the need for systematic supervision to correct the faults detected.

Keywords: Vaccines. Cold chain. Immunization Programs. Community health services.

\section{Introdução}

O desenvolvimento de vacinas seguras e efetivas para a prevenção de doenças infecciosas, geralmente associadas com alta mortalidade e morbidade, é uma das mais significantes realizações na medicina.

Para o êxito dos programas de vacinação, de nada adianta a segurança e eficácia dos imunobiológicos se os profissionais de saúde não os utilizarem ou a população não aderir à vacinação. A literatura aborda com freqüência este assunto crítico, discutindo estratégias para ampliação das coberturas vacinais, ampliando pesquisas sobre imunogenicidade, reatogenicidade e eficácia dos imunobiológicos. No entanto, um fator que pode comprometer a efetividade é relatado com menor freqüência: - como as vacinas são manuseadas durante o transporte e estocagem ${ }^{1}$.

A manutenção da integridade da Rede de Frio $(\mathrm{RF})$ - processo de armazenamento, conservação, distribuição, transporte e manuseio dos imunobiológicos utilizados nos Programas de Imunizações, com o objetivo final de assegurar que todos os produtos administrados mantenham suas características imunogênicas - é um desafio que demanda a completa integração entre os diversos níveis, exigindo compromisso e responsabilidade do Ministério da Saúde e das equipes dos serviços de imunizações.

As falhas no cumprimento das recomendações para a conservação de vacinas na instância local têm sido mais freqüentes do que realmente se acredita, não só em países em desenvolvimento, mas também nos desenvolvidos. Alguns estudos, que pesquisaram conhecimentos e práticas adotadas em sala de vacinação, podem ilustrar este problema, evidenciando desconhecimento dos profissionais sobre intervalos de temperatura adequados para a conservação, inexistência de termômetros ou monitoramento diário de temperaturas, detecção de exposição freqüente dos produtos a extremos de temperatura $\left(<0^{\circ} \mathrm{C}\right.$ e $>10^{\circ} \mathrm{C}$ ) durante o transporte e o armazenamento, organização inadequada 
dos refrigeradores e não exclusividade dos mesmos para estocar vacinas ${ }^{2-14}$.

Estudos anteriores realizados no Brasil descrevem falhas no cumprimento das recomendações para a conservação, necessidade de supervisão permanente e adequação dos recursos humanos e equipamentos ${ }^{15-18}$. Também há relatos de avaliações de amostras de vacinas coletadas nas unidades de saúde com comprometimento na potência ${ }^{19-21}$.

O Programa Nacional de Imunizações brasileiro - PNI (criado em 1973) é responsável pela normatização dos procedimentos a serem adotados na Rede de Frio, consonantes com orientações das Organizações Pan-americana e Mundial de Saúde. Manuais técnicos publicados e inúmeros treinamentos de recursos humanos orientam as atividades para o gerenciamento de estoque, distribuição, manuseio de imunobiológicos e manutenção de equipamentos e outros materiais ${ }^{22-25}$.

Utilizando os manuais revisados e reeditados em 2001, a Divisão de Imunização/Centro de Vigilância Epidemiológica capacitou enfermeiros para a multiplicação dos treinamentos em todos os 645 municípios do Estado de São Paulo.

Não há dados sobre a avaliação do conhecimento adquirido e da prática adotada nas salas de vacina a partir das capacitações realizadas e, a exemplo dos estudos publicados, é possível que procedimentos normatizados não estejam sendo executados nas instâncias locais, colocando sob risco o controle das doenças.

Considerando que o município de São Paulo (MSP) aplicou em 2004 mais de quatro milhões de doses de vacina em 400 salas públicas (rotina e campanhas), e atende a uma população de quase um milhão de crianças menores de cinco anos, sendo que mais de $90 \%$ recebem a vacinação no setor público ${ }^{26}$, avalia-se a importância da correta conservação dos imunobiológicos pelos profissionais para o êxito do Programa de Imunização.

Sendo assim, este estudo se propõe a avaliar o conhecimento e o cumprimento (procedimentos adotados na prática) das recomendações para RF nas salas de vacina do MSP.

É importante relatar que a capital do estado mantém sob controle as doenças imunopreveníveis, refletindo a efetividade das imunizações realizadas nos últimos trinta anos.

\section{Metodologia}

O município de São Paulo é a capital do Estado de São Paulo, possui 1.509 km², o clima é tropical de altitude e as temperaturas médias atingidas durante o ano variam entre $15,7^{\circ} \mathrm{C}$ a $22,4^{\circ} \mathrm{C}(1980-2001$ - Instituto Astronômico e Geofísico - USP). A população estimada é de 10.679 .760 (estimativa 2004 Fundação SEADE; IBGE - população em $1^{\circ}$ de julho), com taxa de crescimento de $0,8 \%$ ao ano entre 1991-2004 e densidade populacional de 7.077,4 hab/ $\mathrm{km}^{2}$. Dados da Fundação SEADE encaminhados ao Centro de Vigilância Epidemiológica para o ano 2004, com base na sobrevivência de nascidos vivos, estimam residir no município de São Paulo 970.980 crianças menores de cinco anos, sendo 181.280 menores de um ano. A organização administrativa é composta de 31 Subprefeituras, com suas respectivas Coordenadorias de Saúde responsáveis pelas ações de assistência, vigilância sanitária e epidemiológica, recursos humanos e financeiros. A distribuição dos imunobiológicos é descentralizada e está sob a responsabilidade de nove Postos de Armazenamento e Distribuição de Imunobiológicos - PADI, que podem abranger mais de uma Subprefeitura. Estes Postos recebem os imunobiológicos diretamente da RF estadual por ainda estar em construção a central de armazenamento municipal.

A partir do cadastro de unidades do Sistema de Informação e Avaliação do Programa de Imunizações (SI-PNI/API) foi obtida a listagem de 390 salas públicas com vacinação de rotina, sob gestão da Secretaria Municipal de Saúde - SMS - em 2003 (excluídas as salas localizadas em hospitais, pronto atendimento e sem produção). 
Um formulário padronizado, com total de dez questões de múltipla escolha, sobre o monitoramento de temperatura, organização interna do refrigerador e conhecimento sobre produtos estáveis/não estáveis a baixas temperaturas foi enviado a todas as unidades. Uma carta endereçada ao diretor da unidade continha esclarecimentos sobre o objetivo da pesquisa e orientações para o preenchimento por dois profissionais da sala de vacina (nível superior e nível médio de formação). $\mathrm{Na}$ ausência de enfermeiro, o responsável pela sala de vacina poderia responder. Também foi enviado um envelope selado para o retorno via postal.

Com as informações sobre doses aplicadas nestas unidades (SI-PNI/API - janeiro a outubro/2003) foi possível distribuí-las em ordem decrescente de produção e agrupá-las em quatro intervalos de classe iguais (quartis). Admitindo-se que $80 \%$ das unidades apresentassem conservação de imunobiológicos adequada com $8 \%$ de margem de erro nesta estimativa, calculou-se uma amostra de 80 unidades para uma visita. A partir dos quatro estratos, sortearam-se 20 unidades de cada um, de maneira aleatória simples. Os 80 estabelecimentos selecionados para a amostra foram visitados para uma entrevista com os profissionais que exercem atividades de vacinação e também para uma observação do equipamento(s) refrigerado(s) utilizado(s) para o armazenamento local de imunobiológicos. Foram utilizados roteiros padronizados e pré-testados. Para a visita aos estabelecimentos foi necessária, por exigência administrativa, a autorização das Coordenadorias de Saúde regionais e das Diretorias de cada unidade. A direção da Unidade definiu o profissional que responderia as questões da entrevista e acompanharia a vistoria do refrigerador. As visitas foram executadas por um grupo de onze pesquisadores (dez enfermeiros e a autora), técnicos com experiência em supervisão e capacitação de pessoal em sala de vacina, com o regis- tro da temperatura de cada geladeira (momento, máxima e mínima) obtido na leitura dos termômetros da unidade. A entrevista foi realizada após a leitura e assinatura do Termo de Consentimento Informado pelo profissional da sala.

O estudo foi conduzido no período de março a junho de 2004.

Um banco de dados foi criado para consolidar as informações coletadas pelos três instrumentos, utilizando-se os programas EPI-INFO Windows, EPI6 - DOS e SPSS V 11.0 para as análises.

Um total de 397 formulários recebidos foi considerado na análise e pertence a 231 unidades públicas de saúde, representando 59,2\% (231/390) do universo amostral (mais do que um formulário por unidade). Todas as 80 unidades selecionadas foram visitadas, porém em duas não havia atividade de vacinação naquele momento e as geladeiras não continham imunobiológicos (uma das unidades em reforma e a outra unidade tinha atividade de vacinação de rotina somente em alguns períodos durante o ano). Nas demais unidades foi possível a observação das geladeiras, sendo que 11 apresentavam mais do que um equipamento para a conservação. Em duas unidades com mais do que uma geladeira havia equipamentos (um em cada unidade) em procedimentos de limpeza e, por estarem vazias, não foram consideradas na análise. Portanto, apesar de 94 refrigeradores comporem o total de equipamentos existentes, 90 serão considerados na análise.

Critérios para a avaliação: Para resumir a avaliação sobre o conhecimento e cumprimento das recomendações do PNI para a RF no MSP, um indicador foi construído para sintetizar os resultados de cada instrumento utilizado. $\mathrm{O}$ indicador foi constituído por pontos atribuídos às respostas dos formulários da seguinte maneira:

- Formulário - Um ponto foi atribuído para cada resposta totalmente correta (concordância com o Manual de Rede 
de Frio/Programa Nacional de Imunizações).

- Entrevista e Observação - a mesma graduação foi atribuída àquelas questões similares ao formulário e que refletissem a execução do conhecimento assinalado, de maneira que fosse possível comparar os resultados obtidos pelos diferentes instrumentos. Por se tratar de constatação direta da prática, a pontuação da observação foi considerada como padrão dos resultados obtidos para cada unidade.

De acordo com a pontuação atingida (máximo de dez pontos) classificou-se arbitrariamente cada instrumento como conhecimento/cumprimento SUFICIENTE (9 a 10), REGULAR ( 7 a 8 ) e INSUFICIENTE $(\leq 6)$.

Os resultados obtidos foram analisados por categoria profissional, tempo de formação, tempo de atividade em sala de vacina e treinamento de sala de vacina. Os testes do $\chi^{2}, \chi^{2}$ de tendência, teste de Fisher, coeficiente de correlação intraclasses (ICC), método de Deyo e medida de concordância de Kappa foram utilizados para as análises.

O projeto foi aprovado pelo Comitê de Ética em Pesquisa da Secretaria Municipal de Saúde, Prefeitura do MSP, sob o número 170 (março/2004). Houve apoio da Coordenadoria de Controle de Doenças da Secretaria de Estado da Saúde de São Paulo para a aquisição de materiais de escritório e deslocamento dos pesquisadores.

\section{Resultados}

A distribuição das 231 unidades respondedoras por estrato de produção (quartis) variou entre $52 \%$ e $67 \%$ e foi considerada homogênea $(p=0,181)$.

Dentre os 397 respondedores do formulário, 98,8\% (392) são profissionais de enfermagem, sendo $47,9 \%$ (190) enfermeiros e 50,9\% (202) auxiliares ou técnicos de enfermagem. Dois formulários foram respondidos por outra categoria profissional (um médico e um gerente) e três não os identificaram. Todos os participantes da entrevista pertenciam à área de enfermagem, 47,5\% (38) enfermeiros e 52,6\% (42) auxiliares/técnicos enfermagem, ressaltando-se que, com a exigência administrativa de pré-agendamento, a definição do profissional a ser entrevistado foi determinada pela diretoria da unidade, o que pode ter influenciado nas características desta população.

O tempo médio de formação dos profissionais de enfermagem (formulário e entrevista) é de 14 a 15 anos. Quarenta (10,2\%) formulários não informaram este dado. Os enfermeiros que responderam o formulário apresentam médias mais elevadas (17 anos) enquanto entre os auxiliares/técnicos o maior número de profissionais está com 5-14 anos de formação, com média de 12,7 anos ( $p=0,000$ ). Esta diferença não foi observada entre os entrevistados.

O tempo médio de atividade em sala de vacina para total de respondedores do formulário é de oito anos (máximo de 30 anos, e 16 profissionais afirmaram exercer trabalho em sala de vacina há menos de um ano), sendo que $11,7 \%$ (46) não informaram este período. Observa-se distribuição semelhante entre enfermeiros e auxiliares/ técnicos. Entre os 38 enfermeiros entrevistados, há predominância no grupo com menos de cinco anos de atividade em sala de vacina $(23=60,5 \%)$, enquanto entre os 42 auxiliares/técnicos, 71,4\% (30) apresentam maior tempo de experiência $(p=0,004)$.

A grande maioria de respondedores $(97,4 \%)$ e entrevistados $(91,3 \%)$ aponta conhecimentos sobre a conservação de imunobiológicos provenientes de capacitação específica em sala de vacina e/ou no curso de graduação. Considerando apenas aqueles que receberam treinamento específico em sala de vacina (formulários - 356/390; entrevistas - 63/ 80), observa-se a tendência de maior proporção de não treinados entre os grupos com menor tempo de atividade em sala de vacina ( $p<0,05$ para $\chi^{2}$ e $\chi^{2}$ tendência) (Tabela 1). Não houve diferença entre os 
Tabela 1 - Distribuição dos profissionais de enfermagem por tempo de atividade em sala de vacina e treinamento, São Paulo/SP, 2004.

Table 1 - Distribution of nursing professionals according to time of activity in vaccine unit and training, São Paulo/SP, 2004.

\begin{tabular}{|c|c|c|c|c|c|c|c|c|}
\hline \multirow{3}{*}{$\begin{array}{l}\text { TEMPO } \\
\text { DE ATIVIDADE } \\
\text { EM SALA DE } \\
\text { VACINA }\end{array}$} & \multicolumn{6}{|c|}{ TREINAMENTO EM SALA DE VACINA(\%) } & \multicolumn{2}{|c|}{ TOTAL(\%) } \\
\hline & \multicolumn{3}{|c|}{ FORMULÁRIO } & \multicolumn{3}{|c|}{ ENTREVISTA } & \multirow{2}{*}{$\begin{array}{l}\text { Form. } \\
(\mathrm{N}=392)\end{array}$} & \multirow{2}{*}{$\begin{array}{l}\text { Entrev } \\
(\mathrm{N}=80)\end{array}$} \\
\hline & SIM & NÃO & OR & SIM & NÃO & OR & & \\
\hline$<5$ anos & $121(34)$ & $20(59)$ & 1,00 & $23(37)$ & $12(71)$ & 1,00 & $141(36)$ & $35(44)$ \\
\hline 5 a 14 anos & $123(35)$ & $6(18)$ & 3,39 & $31(49)$ & $4(24)$ & 4,04 & $129(33)$ & $35(44)$ \\
\hline$>=15$ anos & $73(20)$ & $1(3)$ & 12,07 & $9(14)$ & $1(5)$ & 4,70 & $74(19)$ & $10(12)$ \\
\hline Ignorado & $39(11)$ & $7(20)$ & - & - & - & - & $46(12)$ & - \\
\hline TOTAL & $356(100)$ & $34(100)$ & - & $63(100)$ & 17 (100) & - & $390^{a}(100)$ & $80(100)$ \\
\hline
\end{tabular}

2 profissionais $\mathrm{c} /$ treinamento ignorado/2 professionals with unreported level of training; $\mathrm{OR}=$ odds ratio

enfermeiros e auxiliares/ técnicos de enfermagem e o recebimento de treinamento específico.

Nas visitas verificou-se que o recebimento de vacinas nas unidades é habitualmente mensal em $83,8 \%$ das unidades (67), e as demais com períodos menores (a unidade em reforma não soube responder sobre o transporte e recebimento). Os imunobiológicos vêm acondicionados em caixas térmicas acompanhadas de termômetro em 96,3\% das unidades (77 - uma unidade reformada ainda sem recebimento de vacinas). Todos os entrevistados referem haver uma relação de remessa dos imunobiológicos que é devolvida ao emissor, porém $62,5 \%$ (50/80) não arquivam o registro da temperatura da caixa de transporte na unidade. A maioria $(88,8 \%)$ não refere temperatura de transporte alterada no ano de 2003 (71/80 - um entrevistado não sabe se houve alteração) e 96,3\% (77/80) notificariam esta ocorrência à instância superior se ela acontecesse.

$\mathrm{Na}$ informação dos formulários sobre a marca da geladeira utilizada para a conservação diária de vacinas, 288 indicaram 361 equipamentos, sendo $78,8 \%$ das informantes $(227 / 288)$ com a anotação de um único refrigerador e as demais com dois ou mais. Do total de equipamentos citados, 56\% (202/361) referem-se a refrigeradores específicos para a conservação de vacinas, comercializados pela indústria médico-hospitalar, e 44\% (159/361) são geladeiras comuns ou domésticas. Durante as visitas foram encontradas 94 geladeiras, sendo a proporção entre geladeiras comuns e especiais semelhante - 51\%(48) e $49 \%(46)$. Sessenta e nove unidades visitadas (86\%) dispõem de um único refrigerador; as restantes contam com mais de um equipamento. Uma unidade dispunha apenas de um equipamento para uso diário, sem outra geladeira para estoque.

Os refrigeradores especiais para vacinas correspondem a produtos para uso diário e estoque (capacidade superior a 280 - 300 litros), ou somente para uso diário. O refrigerador especial para uso diário corresponde a equipamento com menor capacidade (cerca de 22 a 30 litros), abertura horizontal, utilizado para armazenar as vacinas a serem utilizadas na jornada de trabalho. No início dos anos 80, um modelo especial da Gelomatic $\AA^{\circledR}$ - chamado Medinic - foi adquirido para os Centros de Saúde pela Secretaria Estadual e até os dias de hoje este tipo de geladeira para uso diário, mesmo que de outros fabricantes, é conhecido pela equipe de enfermagem como "medinic".

Nas visitas, os refrigeradores tipo "medinic" (uso diário) não foram submetidos à observação, exceto em uma unidade por não haver outro equipamento. 
Durante a observação das geladeiras, verificou-se que a média do tempo de uso é de cinco anos (mínimo de um ano e máximo de 20 anos), 43,3\% (39) têm entre um e dois anos de uso, 35,6\% (32) 3 a 10 anos e 14,4\%(13) mais de 11 anos. Seis entrevistados não sabiam referir o tempo de uso dos refrigeradores. A distribuição do tempo de uso de acordo com o tipo de refrigerador aponta que $60,9 \%(28 / 46)$ dos especiais apresentam menos de três anos de uso enquanto 67,3\% (28/44) dos domésticos têm uso por maior tempo do que este $(p=0,007)$. A maioria dos refrigeradores (66/ 90) não apresentou defeitos em 2003 e não houve diferença quanto ao tipo ou tempo de uso do refrigerador.

Entre os itens avaliados sobre conhecimento/cumprimento, as maiores proporções de acerto ocorreram com os procedimentos para o monitoramento de temperatura. A organização do refrigerador doméstico aponta menor proporção de acertos, principalmente no que se refere à colocação de garrafas na parte inferior da geladeira. Quanto aos imunobiológicos e exposições a temperaturas negativas, observa-se a pequena proporção de conhecimento correto para os produtos estáveis. No entanto, são executados procedimentos corretos para a conservação adequada (Tabela 2).

Na observação de refrigeradores especiais ( $\mathrm{N}=45)$, como não há a necessidade de utilizar os artifícios recomendados para a geladeira doméstica, os itens verificados foram a disposição correta das vacinas, permitindo a circulação interna do ar em $66,7 \%$ (30), e a utilização pelo entrevistado dos recursos adicionais para controle: 57,8\% (26) utilizam a temperatura visualizada no painel eletrônico e 46,7 \% (21) sabiam demonstrar as funções do painel.

A classificação das unidades, de acordo com o critério de pontuação estabelecido, permite a avaliação global do conhecimento e da prática, e indica que a pontuação SUFICIENTE foi atribuída a mais da metade dos formulários e entrevistas (53\%

Tabela 2 - Distribuição das respostas corretas para os itens que refletem o conhecimento e prática da conservação de imunobiológicos, São Paulo/SP, 2004.

Table 2 - Distribution of correct answers for items that reflect knowledge and practice on conservation of biologicals, São Paulo/SP, 2004.

\begin{tabular}{|c|c|c|c|c|}
\hline \multirow[t]{2}{*}{ GRUPO } & \multirow[t]{2}{*}{ ÍTEM } & \multicolumn{3}{|c|}{ Respostas corretas } \\
\hline & & $\begin{array}{l}\text { Formulário } \\
\mathrm{N}=397 \text { (\%) }\end{array}$ & $\begin{array}{l}\text { Entrevista } \\
\mathrm{N}=80(\%)\end{array}$ & $\begin{array}{c}\text { Observação } \\
\mathrm{N}=90(\%)\end{array}$ \\
\hline MONITORAMENTO DA & Uso termômetro Max/min & $395(99,5)$ & $80(100)$ & $90(100)$ \\
\hline TEMPERATURA DE & Leitura $\mathrm{T} \geq 2 \mathrm{X} / \mathrm{dia}$ & $396(99,7)$ & $80(100)$ & $88(97,8)$ \\
\hline CONSERVAÇÃO & Impresso para registro & $394(99,2)$ & $79(98,8)$ & $89(98,9)$ \\
\hline \multirow[t]{3}{*}{ (5 pontos) } & Temperatura de conservação adequada & $387(97,5)$ & $80(100)$ & $87(96,7)$ \\
\hline & Notifica alteração de temperatura & $373(94,0)$ & $75(93,8)$ & $80(88,9)^{b}$ \\
\hline & & & & $87(96,7)^{c}$ \\
\hline ORGANIZAÇÃO INTERNA & Congelador com gelo reciclável ${ }^{\mathrm{a}}$ & $115(81,0)$ & $41(100)$ & $35(79,5)$ \\
\hline DO REFRIGERADOR & Parte inferior com garrafas com água ${ }^{a}$ & $98(69,0)$ & $27(65,9)$ & $27(61,4)$ \\
\hline (3 pontos) & Porta vazia ${ }^{a}$ & $127(89,4)$ & $37(90,2)$ & $37(84,1)$ \\
\hline $\begin{array}{l}\text { IMUNOBIOLÓGICOS E } \\
\text { EXPOSIÇÃO A }\end{array}$ & $\begin{array}{l}\text { Imunobiológicos que podem ser } \\
\text { expostos } \leq 0^{\circ} \mathrm{C}\end{array}$ & $73(18,4)$ & $10(12,5)$ & $69(76,7)^{d}$ \\
\hline $\begin{array}{l}\text { TEMPERATURAS NEGATIVAS } \\
\text { ( } 2 \text { pontos) }\end{array}$ & $\begin{array}{l}\text { Imunobiológicos que nunca } \\
\text { podem ser expostos } \leq 0^{\circ} \mathrm{C}\end{array}$ & $322(81,1)$ & $54(67,5)$ & \\
\hline
\end{tabular}

${ }^{a}$ somente geladeiras domésticas/only domestic refrigerators

${ }^{\mathrm{a}} \mathrm{N}$ formulário $=142 / \mathrm{N}$ form $=142 ; \mathrm{N}$ entrevista $=41 /$ interview $=41 ; \mathrm{N}$ observação $=44 / \mathrm{N}$ observation $=44$

${ }^{\mathrm{b}}$ disponibilidade de ficha de notificação/availability of report form

c arquivo mapa mês anterior com notificação de alteração se existente/file with map of previous month and report of changes, if existing

${ }^{\mathrm{d}}$ vacinas corretas na $1^{\mathrm{a}}$ prateleira em domésticas e alarme ativado em especiais/correct vaccines on first shelf of domestic refrigerator and activated alarm in special refrigerator 
Tabela 3 - Distribuição dos conceitos obtidos na OBSERVAÇÃO, ENTREVISTA E FORMULÁRIO - avaliação do conhecimento e prática na conservação de imunobiológicos em sala de vacina, São Paulo/SP, 2004

Table 3 - Distribution of scores attained on OBSERVATION, INTERVIEW AND FORM - assessment of knowledge and practice on conservation of biologicals in vaccine units, São Paulo/SP, 2004.

\begin{tabular}{lcccccc}
\hline PONTUAÇÃO & \multicolumn{2}{c}{ OBSERVAÇÃO } & \multicolumn{2}{c}{ ENTREVISTA } & \multicolumn{2}{c}{ FORMULÁRIO } \\
& $\mathrm{N}$ & $\%$ & $\mathrm{~N}$ & $\%$ & $\mathrm{~N}$ & $\%$ \\
\hline Suficiente & 59 & 66 & 44 & 55 & 209 & 53 \\
Regular & 24 & 27 & 34 & 43 & 176 & 44 \\
Insuficiente & 7 & 7 & 2 & 2 & 12 & 3 \\
TOTAL & 90 & 100 & 80 & 100 & 397 & 100 \\
\hline
\end{tabular}

e 55\%) e a dois terços das observações (66\%) (Tabela 3).

Para as análises, os conceitos REGULAR E INSUFICIENTE foram agrupados devido à proporção deste último apresentar-se reduzida.

Cada refrigerador observado foi contabilizado com a categoria profissional que respondeu a entrevista, respectivo treinamento e tempo de atividade em sala de vacina. Como havia unidades com mais de um refrigerador, calculouse a média da pontuação obtida nos roteiros de observações para a associação com as características dos entrevistados (total de pares $=78$ ).

A distribuição dos conceitos por categoria profissional, tempo de atividade e treinamento em sala de vacina é mostrada na Tabela 4 e entre os profissionais que receberam treinamento específico a proporção de acertos na OBSERVAÇÃO foi significativamente maior $(p=0,009)$. As demais categorias analisadas não apresentam diferenças significativas.

Analisando-se separadamente as categorias profissionais treinadas verifica-se uma diferença significativa entre a pontuação obtida pelos enfermeiros respondedores dos formulários. A diferença entre os respondedores não treinados não apresentou significância estatística (Tabela 5). Entre os entrevistados também não houve diferença nos resultados obtidos de acordo com as categorias profissionais e o tempo de realização do treinamento.

Tabela 4 - Distribuição da pontuação obtida para avaliação da conservação de imunobiológicos, por categoria profissional, treinamento e tempo de atividade em sala, São Paulo/SP, 2004.

Table 4 - Distribution of scores attained for assessment of conservation of biologicals, according to professional category, training and time of activity in vaccine unit, São Paulo/SP, 2004.

\begin{tabular}{|c|c|c|c|c|c|c|}
\hline \multirow[t]{2}{*}{ AVALIAÇÃO } & \multicolumn{2}{|c|}{ FORMULÁRIO (N=397) } & \multicolumn{2}{|c|}{ ENTREVISTA $(\mathrm{N}=80)$} & \multicolumn{2}{|c|}{ OBSERVAÇÃO $(\mathrm{N}=78)^{\mathrm{d}}$} \\
\hline & Sufic. & Reg+Insuf & Sufic. & Reg+Insuf. & Sufic. & Reg+Insuf. \\
\hline \multicolumn{7}{|l|}{ Profissãoa } \\
\hline Enfermeiro & $108(56,8)$ & $82(43,2)$ & $20(52,6)$ & $18(47,4)$ & $26(70,3)$ & $11(29,7)$ \\
\hline Aux/Técnico & $97(48,0)$ & $105(52,0)$ & $24(57,2)$ & $18(42,8)$ & $25(61,0)$ & $16(39,0)$ \\
\hline \multicolumn{7}{|c|}{ Treinamento $^{b}$} \\
\hline $\operatorname{Sim}$ & $189(52,5)$ & $171(47,5)$ & $33(52,4)$ & $30(47,6)$ & $45(72,6)$ & $17(27,4)$ \\
\hline Não & $18(53,0)$ & $16(47,0)$ & $11(64,7)$ & $6(35,3)$ & $6(37,5)$ & $10(62,5)$ \\
\hline \multicolumn{7}{|c|}{ Atividade em salac } \\
\hline$<5$ anos & $71(50,3)$ & $70(49,6)$ & $20(57,1)$ & $15(42,9)$ & $23(65,6)$ & $12(34,4)$ \\
\hline 5 ou + anos & $116(55,8)$ & $92(44,2)$ & $24(53,3)$ & $21(46,7)$ & $28(65,2)$ & $15(34,8)$ \\
\hline
\end{tabular}

a cinco formulários sem informação da profissão/five forms without professional information

${ }^{b}$ três formulários sem informação sobre treinamento/three forms without information on training

c 48 formulários sem informação sobre tempo de atividade em sala de vacina/48 forms without information on time of activity in vaccine unit

${ }^{\mathrm{d}}$ média do conceito da observação pareado com entrevistado/mean of observation score paired with interviewee 
Tabela 5 - Distribuição da pontuação obtida no formulário de avaliação na conservação de imunobiológicos por categoria profissional e treinamento, São Paulo/SP, 2004.

Table 5 - Distribution of scores attained in the assessment form on conservation of biologicals, according to professional category and training, São Paulo/SP, 2004.

\begin{tabular}{|c|c|c|c|c|c|c|c|c|c|c|c|}
\hline \multirow{3}{*}{$\begin{array}{l}\text { PROFISSÃO } \\
\text { Enfermeiro }\end{array}$} & \multicolumn{4}{|c|}{ TREINADO ${ }^{\mathrm{a}}$} & \multicolumn{4}{|c|}{ NÃO TREINADOb } & \multicolumn{3}{|c|}{ TOTAL } \\
\hline & \multicolumn{2}{|c|}{ Suficiente } & \multicolumn{2}{|c|}{ Reg+Insuf } & \multicolumn{2}{|c|}{ Suficiente } & \multicolumn{2}{|c|}{ Reg+Insuf } & \multirow{2}{*}{$\frac{T}{171}$} & \multirow{2}{*}{$\begin{array}{l}\text { NT } \\
18\end{array}$} & \multirow{2}{*}{$\frac{(\%)}{(100,0)}$} \\
\hline & 100 & $58,5 \%$ & 71 & $41,5 \%$ & 7 & $38,9 \%$ & 11 & $61,1 \%$ & & & \\
\hline Aux/técnico & 85 & $45,9 \%$ & 100 & $54,1 \%$ & 11 & $68,7 \%$ & 5 & $31,3 \%$ & 185 & 16 & $(100,0)$ \\
\hline TOTAL & 185 & $51,9 \%$ & 171 & $48,1 \%$ & 18 & $52,9 \%$ & 16 & $47,1 \%$ & 356 & 34 & $(100,0)$ \\
\hline
\end{tabular}

${ }^{\mathrm{a}} \chi 2=5,59 \mathrm{p}=0,018 \quad{ }^{\mathrm{b}}$ Fisher $\mathrm{p}=0,100$

A comparação dos conceitos obtidos entre as observações e as entrevistas, pareados por unidade, indica que $50 \%$ (39/78) obtiveram pontuações iguais. A mesma comparação foi possível entre formulários e observações. Identificaram-se 52 unidades visitadas que enviaram formulários (calculada a média quando havia mais de um formulário por unidade) e as pareadas indicaram que $46,2 \%$ (24) obtiveram pontuações iguais. Os índices de Kappa e os coeficientes de correlação intraclasses (método de Deyo) indicam que a concordância entre os resultados obtidos é baixa. Os resultados semelhantes podem ter sido obtidos ao acaso (Tabelas 6 e 7 ).
No sentido de identificar se a baixa concordância poderia ser atribuída a algum item em especial, analisaram-se separadamente os grupos de questões que representam o monitoramento de temperatura, a organização interna do refrigerador e o conhecimento dos produtos instáveis/estáveis nas temperaturas negativas.

No grupo sobre o monitoramento, a baixa concordância reside principalmente na referência do entrevistado em notificar a alteração de temperatura e no fato de a unidade não dispor de fichas de notificação ou mapas para registro de temperatura. Ou também o inverso, disponibilidade de instrumentos de notifi-

Tabela 6 - Distribuição dos conceitos obtidos nos roteiros de observação (média) e entrevistas, São Paulo/SP, 2004.

Table 6 - Distribution of scores attained in OBSERVATION (mean) and INTERVIEW, São Paulo/SP, 2004.

\begin{tabular}{|c|c|c|c|c|c|c|}
\hline \multirow{3}{*}{$\begin{array}{l}\text { ENTREVISTAS } \\
\text { Suficiente (9 a 10) }\end{array}$} & \multicolumn{6}{|c|}{ OBSERVAÇÕES } \\
\hline & \multicolumn{2}{|c|}{ Suficiente (9 a 10) } & \multicolumn{2}{|c|}{ Insuficiente +Regular $(\leq 8)$} & \multicolumn{2}{|c|}{ TOTAL } \\
\hline & 28 & $54,9 \%$ & 16 & $59,3 \%$ & 44 & $56,4 \%$ \\
\hline Insufic + Regular $(\leq 8)$ & 23 & $45,1 \%$ & 11 & $40,7 \%$ & 34 & $43,6 \%$ \\
\hline TOTAL & 51 & $100,0 \%$ & 27 & $100,0 \%$ & 78 & $100,0 \%$ \\
\hline
\end{tabular}

Kappa $=-0,04 \mathrm{p}=0,644 \quad \mathrm{ICC}=0,05$

Tabela 7 - Distribuição dos conceitos obtidos nos roteiros de observação (média) e formulários das unidades visitadas, São Paulo/SP, 2004.

Table 7 - Distribution of scores attained in OBSERVATION (mean) and FORM of units visited, São Paulo/SP, 2004.

\begin{tabular}{|c|c|c|c|c|c|c|}
\hline \multirow{3}{*}{$\begin{array}{l}\text { FORMULÁRIOS } \\
\text { Suficiente (9 a 10) }\end{array}$} & \multicolumn{6}{|c|}{ OBSERVAÇÕES } \\
\hline & \multicolumn{2}{|c|}{ Suficiente (9 a 10) } & \multicolumn{2}{|c|}{ Insuficiente +Regular $(\leq 8)$} & \multicolumn{2}{|c|}{ TOTAL } \\
\hline & 14 & $43,8 \%$ & 10 & $50,0 \%$ & 24 & $56,4 \%$ \\
\hline Regular (7-8) & 18 & $56,3 \%$ & 10 & $50,0 \%$ & 28 & $43,6 \%$ \\
\hline TOTAL & 32 & $100,0 \%$ & 20 & $100,0 \%$ & 52 & $100,0 \%$ \\
\hline
\end{tabular}

Kappa $=-0,06 \quad \mathrm{p}=0,670 \quad$ ICC $=-0,09$ 
cação e monitoramento, e não conhecimento do entrevistado deste procedimento.

No grupo referente à estabilidade de produtos às baixas temperaturas, observase baixo índice de conhecimento, porém a execução dos procedimentos que evitam esta exposição é paradoxalmente maior.

Quanto à organização da geladeira ocorreu a maior concordância: procedimentos executados de acordo com os referidos no conhecimento com maior freqüência (Figura 1) (Карpa=0,53 e p=0,000).

\section{Discussão}

Este estudo apresentou o retorno espontâneo de 397 formulários correspondentes a quase $60 \%$ das unidades (231/ 390), taxa superior a estudo semelhante realizado em Sidney $(1992)^{7}$ e inferior a estudos que apresentaram universo menor de unidades ou controlaram o envio das respostas ${ }^{6,12}$. Todas as unidades selecionadas na amostra foram visitadas.

A homogeneidade dentre as unidades respondedoras, quanto ao estrato de produção, poderá minimizar diferenças nos resultados apresentados se as nãorespondedoras participassem do estudo. Ressalte-se que a estratificação pela produção de doses aplicadas foi o critério adotado para eleger as unidades visitadas visando uniformizar a seleção.

A participação maciça dos profissionais de enfermagem (respondedores e entrevistados), também referenciada em outros estudos brasileiros ${ }^{15-18}$, confirma que a responsabilidade pela conservação de vacinas no setor público é atribuição desta categoria profissional. Vale ressaltar que a lei de exercício profissional brasileira permite a administração de vacinas por profissionais de enfermagem, médicos e farmacêuticos. Associado ao elevado percentual de profissionais que receberam treinamento em sala de vacina (70 a 90\%), evidencia-se que o recurso humano no MSP tem formação técnica adequada para exercer atividades específicas da sala de vacina.

A definição pela diretoria da unidade do profissional que seria entrevistado pode ter interferido nas características desta população. Observa-se que os auxiliares/técnicos de enfermagem tinham maior tempo de atividade em sala de vacina, comparados aos enfermeiros, e possivelmente esta maior experiência pode ter sido o critério de escolha quando o enfermeiro não estava à disposição para a visita. Por outro lado, não houve diferença entre estas categorias e o recebimento de treinamento específico,

\section{ORGANIZAÇÃO DO REFRIGERADOR}

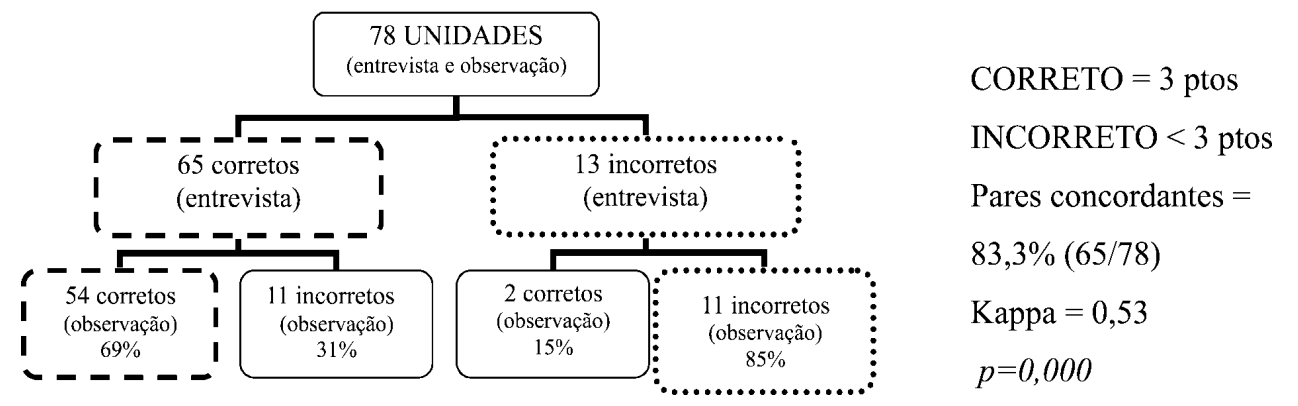

Figura 1 - Concordância entre as respostas obtidas nas ENTREVISTAS e OBSERVAÇÕES avaliação do conhecimento e prática na organização do refrigerador para vacinas, São Paulo/SP, 2004.

Figure 1 - Agreement between answers obtained in INTERVIEW and OBSERVATION assessment of knowledge and practice in organization of vaccine refrigerator, São Paulo/SP, 2004. 
presumindo-se que as capacitações são abrangentes.

$\mathrm{O}$ achado de geladeiras especiais para a conservação de vacinas em parcela importante das unidades e com pouco tempo de uso (menos de três anos) indica que existe uma preocupação recente em fornecer equipamentos tecnologicamente mais avançados e melhorar a qualidade da RF nas unidades do MSP. No entanto, o despreparo das equipes locais no manuseio destes refrigeradores e a subutilização de seus acessórios pode acarretar prejuízos no investimento realizado (redução no custo-benefício) e, o que é pior, colocar sob risco a qualidade dos imunobiológicos ofertados à população.

Vale lembrar que o Manual de RF do $\mathrm{PNI}^{22}$ faz somente recomendações sobre a organização interna de geladeiras domésticas para armazenagem em sala de vacina e, inicialmente, pensou-se que este fator pudesse ter gerado a dificuldade em responder os formulários em relação a estes itens. No entanto, durante as entrevistas foi possível obter respostas mais direcionadas sobre o conhecimento da organização dos diferentes tipos de geladeira, e os resultados (Tabela 2) dos itens de organização das geladeiras domésticas se repetem. A organização correta concomitantemente nos três compartimentos (congelador, "piso" e porta) foi indicada por $68,2 \%$ dos 41 entrevistados que tinham geladeira doméstica em suas unidades. Seria importante que, em futuras revisões e edições dos Manuais de RF, fossem incluídas orientações sobre o uso de geladeiras específicas para o armazenamento de vacinas, freqüentes no setor privado (supervisionado pelo gestor local), e em número crescente no setor público.

Falhas no cumprimento da organização da geladeira, principalmente o uso de garrafas com água na última prateleira e a não exclusividade do refrigerador para vacinas também são referidas em outros estudos 2,3,5,9,13,15-18, 20, 21. No estudo do MSP não foram encontrados alimentos, espécimes laboratoriais ou medicamentos em nenhum refrigerador e, não obstante o pré-agendamento das visitas possa ter interferido nestes resultados, destaque-se que a maior concordância entre o conhecimento e a prática ocorreu nos itens de organização do refrigerador.

Os estudos mais recentes sobre RF, além de evidenciar a falta de cuidados na manutenção dos imunobiológicos sob temperaturas ótimas (entre 2 e $8^{\circ} \mathrm{C}$ ), têm ressaltado a falta de conhecimento, entre os responsáveis pelas ações de imunização, dos prejuízos acarretados pelas baixas temperaturas ${ }^{5,9,27}$. No MSP, grande parte dos profissionais tem a consciência deste risco ( $81 \%$ respondedores e $68 \%$ entrevistados) e $77 \%$ dos refrigeradores observados têm organização correta, protegendo contra exposições a temperaturas próximas de zero (alarme ativado em geladeiras especiais e vacinas sensíveis abaixo da primeira prateleira em geladeiras domésticas - Tabela 2).

O baixo índice de conhecimento sobre as vacinas que podem ser congeladas $(18,4 \%$ dos respondedores e $12,5 \%$ dos entrevistados) não coloca sob risco a efetividade do Programa, pois nas unidades de saúde não existem freezers, não se armazenam vacinas no congelador e todos os profissionais conhecem os limites de temperatura de conservação. A conduta mais adequada, constatada no MSP, é a de manter qualquer imunobiológico da instância local sob temperatura entre $2^{\circ}$ e $8^{\circ} \mathrm{C}$, de toda e qualquer alteração de temperatura (fora destes limites) ser registrada, comunicada, e de os produtos não serem utilizados sem autorização expressa da coordenação central.

A leitura das temperaturas de momento, máxima e mínima, realizada no termômetro existente na unidade, não encontrou nenhum valor abaixo de $0^{\circ} \mathrm{C}$. Duas geladeiras tinham temperatura máxima acima de $8^{\circ} \mathrm{C}$ (a maior temperatura encon- 
trada foi de $23^{\circ} \mathrm{C}$ ). Em $97 \%$ das geladeiras (87), a temperatura no momento estava entre $2^{\circ}$ e $8^{\circ} \mathrm{C}$, e nos outros três equipamentos foram encontrados registros entre $0^{\circ} \mathrm{C}$ e $2^{\circ} \mathrm{C}$. Não há diferença estatística entre os tipos de refrigeradores (especiais ou domésticos) que apresentaram temperaturas alteradas nas visitas.

$\mathrm{O}$ encontro de temperaturas adequadas no momento da visita foi considerado elevado, semelhante ao descrito por Molina e cols. ${ }^{27}$, e melhores do que o de outros estudos, mesmo em países desenvolvidos $^{5,6,9,12,19}$. Ressalta-se que este estudo tem a limitação de avaliar a temperatura em um único ponto, e não se utilizou instrumento previamente aferido para sua mensuração, mas apenas a leitura do termômetro disponível na unidade. Apesar da reduzida identificação de temperaturas inadequadas, deve-se considerar que foi observada falha em itens diversos e a não adesão a todos os procedimentos normatizados não garantirá a manutenção da correta estocagem, podendo acumular incorreções com conseqüências inaceitáveis.

A baixa concordância entre as graduações totais obtidas com os instrumentos utilizados (formulário, entrevista e observação) induz à reflexão sobre a forma como as informações nos treinamentos são transmitidas. A maior concordância para o grupo de questões sobre a organização da geladeira reflete a relevância da parte prática dos treinamentos, sendo necessária a adoção de técnicas que proporcionem aos treinandos maiores possibilidades de apreensão dos "por quês" da execução de cada procedimento. Parece indispensável o acompanhamento dos profissionais treinados nas unidades para dar sustentabilidade às capacitações realizadas.

A estrutura das unidades públicas de saúde do MSP para a conservação de vacinas está adequada, uma vez que há equipamentos refrigerados e termômetros em todas, o abastecimento de vacinas tem periodicidade adequada e regular, o transporte é realizado em caixas térmicas monitoradas com termômetros, e os recursos humanos têm formação para exercer atividades específicas do programa de imunização (profissionais de saúde graduados em enfermagem). A capacitação específica para as atividades em sala de vacina foi abrangente, principalmente nos últimos cinco anos, atingindo a maioria dos profissionais, sem distinção de categorias (enfermeiros X auxiliares/técnicos). Os treinamentos realizados influenciaram positivamente na correta execução dos procedimentos necessários para a conservação de imunobiológicos, embora a sedimentação dos conhecimentos teóricos que suportam a prática ainda necessite de maior ênfase. A grande maioria dos profissionais conhece a importância da utilização do termômetro de máxima e mínima, a leitura diária de temperaturas, o registro em impresso próprio e a notificação das alterações ao nível hierárquico superior.

O Programa de Imunização em São Paulo, semelhante aos demais municípios brasileiros, é fundamentalmente exercido pelos profissionais de enfermagem e a supervisão da assistência deve ser exercida pelo enfermeiro. Trabalhos anteriores desenvolvidos no Estado de São Paulo apontam a importância da supervisão sistemática, não só contemplando aspectos organizacionais e educativos, mas também para a melhoria na humanização do atendimento em sala de vacina $^{16-18}$. Este estudo aponta para a importância da presença do supervisor periodicamente em todas as salas de sua área de abrangência, uma vez que as informações fornecidas pelos profissionais (escritas ou verbais - formulários e entrevistas) não são absolutamente concordantes com a prática.

Finalmente, é necessário instruir as unidades que, por ocasião do recebimento de novos equipamentos específicos para a conservação de vacinas, solicitem a instalação, com demonstração da sua utilização por parte do fabricante, sempre supervisi- 
onada pelas equipes regionais. Recomenda-se que o manual técnico instrucional que acompanha cada equipamento permaneça na sala de vacina.

É indiscutível que a constatação do procedimento executado corretamente através da observação direta da organização de cada sala fornece subsídios primorosos para a avaliação global da RF do MSP. Os resultados aqui apresentados, não reprodutíveis para $100 \%$ das unidades públicas municipais, configuram cenário de certa forma satisfatório e as falhas detectadas, como já foi relatado, são de fácil resolução.

\section{Agradecimentos}

O primeiro autor agradece a colaboração e participação dos enfermeiros que executaram as visitas às unidades, às equipes central e regionais de coordenação do Programa de Imunização no MSP e aos profissionais que colaboraram voluntariamente respondendo à pesquisa.

\section{Referências}

1. Casto DT, Brunell PA. Safe Handling of Vaccines. Pediatrics 1991; 87: 108-12.

2. Carrasco R, Dinstrans R, Montaldo I, Medina E, Reyes M, Vergara I e cols. Cadena de frio del Programa Ampliado de Inmunización - Una experiencia de evaluacion. Boletin de la Oficina Sanitaria Panamericana 1983; 94(1):37-45.

3. Roos LR, Ibarra CF, Pacheco GL. Evaluacion mediante acreditacion de la cadena de frio del Programa de Inmunizaciones del Servicio de Salud Metropolitano Ocidente. Bol Hospital “San Juan de Dios” 1984; 31: 370-3.

4. Lugosi L, Battersby A. Transport and storage of vaccines in Hungary: the first cold chain monitor study in Europe. Bull World Health Organ 1990: 68(4): 431-9.

5. Bishai DM, Bhatt S, Miller LT, Hayden GF. Vaccine storage pratices in pediatric offices. Pediatrics 1992; 89: 193-6.

6. Thakker Y, Woods S. Storage of vaccines in the community: weak link in the cold chain? BMJ 1992; 304: 756-8.

7. Rixon G, Lyn March, Holt DA. Immunisation practices of general practitioners in metropolitan Sydney. Aust J Public Health 1994; 18(3): 258-60.

8. Miller NC, Harris MF. Are childhood immunization programmes in Australia at risk? Investigation of cold chain in the Northern territory. Bull World Health Organ 1994: 72(3): 401-8.

9. Yuan L, Stacy D, Naus M, Branimir B. Vaccine storage and handling: knowledge and practice in primary care physician' offices. Can Fam Physician 1995; 41: 1169-76.

10. Oncel S, Dallar Y, Tanyer G. Evaluation of vaccine storage in Turkey. World Health Forum 996, 17: 287-8.

11. Warwryk A, Mavromatis C, Gold M. Eletronic monitoring of vaccine cold chain in a metropolitan area. BMJ 1997; 315: 518.
12. Grasso M, Ripabelli G, Sammarco ML, Selvaggi TMM, Quaranta A. Vaccine storage in the community: a study in central Italy. Bull World Health Organ 1999; 77(4): 352-4.

13. Bell KN, Hogue CJR, Manning C, Kendal AP. Risk Factors for improper vaccine storage and handling in private provider offices. Pediatrics 2001; 107: e100.

14. Lewis PR, Reimer RF, Dixon AJ. Evaluating the efficacy of vaccine storage in the general practice setting. Aust NZJ Public Health 2001; 25(6): 547-50.

15. Lima GZ, Baldy JLS, Souza MSO. Uso de refrigeradores para conservação de vacinas nas unidades de saúde do município de Londrina, Paraná. Pediatria (São Paulo) 1985; $7: 17-9$.

16. Gonçalves ML. Programa de Vacinação no processo de municipalização da saúde, Ribeirão Preto [dissertação de mestrado]. Ribeirão Preto: Universidade de São Paulo; 1994.

17. Silva EC. Avaliação do sistema de cadeia de frio e do processo de aplicação de vacinas em quatro unidades básicas de saúde [dissertação de mestrado]. São Paulo: Escola Paulista de Medicina; 1993.

18. Escobar EMA. Avaliação da qualidade do Programa de Imunização do município de Vinhedo/SP [tese de doutorado]. São Paulo: Universidade Federal de São Paulo; 2000.

19. Mendes IF, Pral MM, Miyaki C, Gallina NMF, Petricevich VL, Fang FLW et al. Avaliação das condições de estocagem de vacinas vivas, atenuadas contra sarampo, em postos de vacinação credenciados e em Centros de Saúde do estado de São Paulo. Rev Saúde Publica 1985; 19: 444-9.

20. Oliveira SA, Loureiro MLP, Kiffer CRV, Maduro LMF. Reevaluation of the basic procedures involved in the storage of measles vaccine in public health units of the municipality if Niteroi, state of Rio de Janeiro, Brazil. Rev Soc Bras Med Trop 1993; 26(3): 145-9. 
21. Oliveira SA, Homma A, Mahul DC, Loureiro MLP Camillo-Coura L. Avaliação das condições de estocagem de vacina contra o sarampo nas unidades sanitárias dos municípios de Niterói e São Gonçalo, estado do Rio de Janeiro. Rev Inst Med Trop São Paulo 1991; 33(4): 313-8.

22. Fundação Nacional de Saúde. Programa Nacional de Imunizações. Manual de Rede de Frio. $3^{\text {a }}$ edição. Brasília: Ministério da Saúde; 2001.

23. Fundação Nacional de Saúde. Programa Nacional de Imunizações. Capacitação de Pessoal em Sala de Vacinação - manual do treinando. Brasília: Ministério da Saúde; 2001.

24. Fundação Nacional de Saúde. Programa Nacional de Imunizações. Capacitação de Pessoal em Sala de Vacinação - manual do monitor. Brasília: Ministério da Saúde; 2001.
25. Secretaria de Ações Básicas de Saúde. Textos Básicos de Saúde. Programa Nacional de Imunizações. Rede de Frio: noções básicas de refrigeração e procedimentos para a conservação de imunobiológicos. Brasilia: Ministério da Saúde; 1988.

26. Centro de Estudos Augusto Leopoldo Ayrosa Galvão. Inquérito de Cobertura Vacinal do Município de São Paulo. São Paulo: Faculdade de Ciências Médicas da Santa Casa de São Paulo; 2002.

27. Molina PO, Arbiza PA, Vicente RA, Rábago MLG, Pardo JRJ, Rojas VD. Cadena del frío para la conservación de las vacunas en los centros de atención primaria de una área de Madrid; Mantenimiento y nivel de conocimientos. Rev Esp Salud Publica 2002; 76(4): 333 46

Recebido em: 09/08/05 Versão reformulada reapresentada em: 03/02/06 Aprovado em: 30/03/06 